\title{
Collapsing regions and black hole formation
}

\author{
Gregory A. Burnett \\ Department of Physics, University of Florida, Gainesville, Florida 32611
}

(4 August 1995)

\begin{abstract}
Up to a conjecture in Riemannian geometry, we significantly strengthen a recent theorem of Eardley by proving that a compact region in an initial data surface that is collapsing sufficiently fast in comparison to its surface-to-volume ratio must contain a future trapped region. In addition to establishing this stronger result, the geometrical argument used does not require any asymptotic or energy conditions on the initial data. It follows that if such a region can be found in an asymptotically flat Cauchy surface of a spacetime satisfying the null-convergence condition, the spacetime must contain a black hole with the future trapped region therein. Further, up to another conjecture, we prove a strengthened version of our theorem by arguing that if a certain function (defined on the collection of compact subsets of the initial data surface that are themselves three-dimensional manifolds with boundary) is not strictly positive, then the initial data surface must contain a future trapped region. As a byproduct of this work, we offer a slightly generalized notion of a future trapped region as well as a new proof that future trapped regions lie within the black hole region.
\end{abstract}

04.20.Dw, 04.70.Bw, 04.20.-q

\section{INTRODUCTION}

Given an initial data set for the gravitational field $\left(\Sigma, g_{a b}, K_{a b}\right)$ [1] associated with a Cauchy surface in an asymptotically flat spacetime, can we tell whether gravitational collapse has proceeded to such a point that a black hole has formed in that spacetime? In principle, the answer is yes: Given a complete description of the matter fields in the spacetime (i.e., their initial data and evolution equations), then using Einstein's equation, evolve the initial data to reconstruct the entire spacetime and then see whether the spacetime has a nonempty blackhole region and, if so, whether and where it intersects $\Sigma$. In practice, however, carrying out this construction is a highly nontrivial task, even in the vacuum case and even when done numerically [2].

While there is currently no simple algorithm for determining from an initial data set whether a black hole has formed, if a future trapped region exists in $\Sigma$, it must lie within the black-hole region, provided the spacetime satisfies the null-convergence condition [3, [1,5]. Recall that a closed subset $C$ of $\Sigma$ having the structure of a three-manifold with smooth (or at least $C^{2}$ ) boundary, bounded away from spatial infinity, is said to be a future trapped region if the convergence of the future-directed null geodesics orthogonal to $\partial C$ and outward directed (in the sense that the projection of null geodesic tangent vectors on $C$ into $\Sigma$ point outward from $C$ ) is non-negative everywhere on $\partial C$ [ $[$ ]. Denoting the induced metric on $\partial C$ by $h_{a b}$, the mean extrinsic curvature [6] of $\partial C$ in $\Sigma$ by $H$, and the extrinsic curvature of $\Sigma$ in the spacetime by $K_{a b}, C$ is a future trapped region if

$$
H \leq K_{a b} h^{a b}
$$

on $\partial C$. Likewise, the total future trapped region of $\Sigma$ (being the closure of the union of all future trapped regions in $\Sigma$ ) along with its boundary $\mathcal{A}$ (the future apparent horizon, on which $\left.H=K_{a b} h^{a b}\right)$, is contained within the black-hole region (under the same conditions as before) [7]. So, in initial data sets with a non-empty total future trapped region, gravitational collapse has proceeded sufficiently far so that black holes have formed.

Are there any simple conditions that guarantee the existence of a future trapped region in an initial data set? Thorne's "hoop conjecture" offers a test of this type: If a body of mass $M$ is sufficiently compact so that a hoop of circumference $4 \pi M$ can encircle the body no matter how it is rotated there about, then the body must be contained within a horizon [8,9]. While a precise version of this conjecture remains to be proven, Schoen and Yau have proven that an initial data set containing a region $\Omega$ with sufficient matter density must contain a future or past apparent horizon [10]. This interesting result has the slight weaknesses that its requirement on the matter content is so strict that arbitrarily small vacuum regions in $\Omega$ are not allowed and that, as a time-symmetric theorem, we cannot conclude that the apparent horizon must be a future horizon. (Of course, this last criticism can be avoided by restricting oneself to initial data sets that do not contain past trapped regions.) Further, a number of sufficient conditions have been found for spherically symmetric initial data sets [11]. More recently, using Jang's equation and its properties as established by Schoen and Yau [12], Eardley has recently provided a remarkably simple proof of the following theorem 13, 14.

Theorem (Eardley). Fix an asymptotically flat initial data set for the gravitational field $\left(\Sigma, g_{a b}, K_{a b}\right)$ [1] satisfying the dominant-energy condition. If there exists a compact region $\Omega \subset \Sigma$ such that $K_{a b}\left(g^{a b}-n^{a} n^{b}\right)$ is no less than the surface-to-volume ratio of $\Omega$ for all unit vectors $n^{a}$ everywhere on $\Omega$, then $\Sigma$ must contain an apparent horizon.

Were the apparent horizon a future apparent horizon, then, as noted, the conditions of this theorem would 
guarantee that gravitational collapse has proceeded sufficiently far that a black-hole region has formed. Unfortunately, as given, the theorem alone does not allow one to draw this conclusion as it suffers from the same problem of Schoen and Yau's theorem [10] in that the possibility that that all such horizons will be past apparent horizons has not been eliminated. However, the time-asymmetry in the hypotheses of Eardley's theorem $\left(K^{a}{ }_{a}\right.$ is strictly positive on $\Omega$ indicating that the region is collapsing "on average") is a strong indication that there should be a future apparent horizon somewhere in $\Sigma$.

By changing our viewpoint, Eardley's theorem suggests an alternative argument having the advantage of producing a strengthened version of the above theorem under weaker hypotheses. In particular, we can now show that $\Sigma$ must in fact contain a future apparent horizon. Further, this argument has an entirely geometric character, which is to be compared to Eardley's argument, which, through the use of Jang's equation, has an analytic character.

To begin, notice that the induced metric $h^{a b}$ on a twosurface $\mathcal{S} \subset \Sigma$ can be written as $h^{a b}=g^{a b}-n^{a} n^{b}$, where $n^{a}$ is either of the two unit-normal vectors to $\mathcal{S}$. Therefore, the hypothesis of the above theorem guarantees that, on $\mathcal{S}, K_{a b} h^{a b}$ is bounded from below by the surfaceto-volume ratio of $\Omega$, which we denote by $\sigma(\Omega)$. Notice that this bound is independent of the two-surface in $\Omega$. This suggests that if there exists a region in $\Omega$ (having the structure of a three-manifold with boundary) whose boundary's mean extrinsic curvature $H$ is bounded above by $\sigma(\Omega)$, then Eq. (1.1) would hold on the boundary, and hence the region would be a future trapped region.

Does such a region exist in $\Omega$ ? The appearance of the surface-to-volume ratio $\sigma$ in Eardley's theorem suggests that we study this quantity as function on the collection of regions $C$ in $\Omega$. Consider a region $C \subset \Omega$ that is "nearly degenerate" in the sense that it is either flat like a pancake of thickness $r$, thin like a cigar of radius $r$, or small like a sphere of radius $r$. Then, we expect (as in the flat space case) that $\sigma(C) \approx$ const $/ r$, for $r$ sufficiently small, showing that regions that are nearly degenerate in the sense that they are small in one or more dimensions have very large surface-to-volume ratios. This suggests that there is some sufficiently well-behaved region in $\Omega$ having minimal surface-to-volume ratio. In fact, we conjecture that there always exists a region $\hat{C} \subset \Omega$, having the structure of a differentiable manifold with boundary, that minimizes $\sigma$ over such regions $C \subset \Omega$. (See conjecture 1 in Sec. IV.) Remarkably, it then follows that $H \leq \sigma(\Omega)$ on any open subset of $\partial \hat{C}$ where the surface is $C^{2}$, i.e., on the portion having a well-defined and continuous extrinsic curvature. (The proof of this fact is given in Sec. II.)

Putting this all together, we have

$$
H \leq \sigma(\Omega) \leq K_{a b} h^{a b}
$$

on the open subset of $\partial \hat{C}$ that is $C^{2}$, where the first in- equality is a consequence of the minimizing property of $\hat{C}$ and the second follows by hypothesis. Were $\partial \hat{C}$ everywhere $C^{2}$, then $\hat{C}$ would be a future trapped region. As explained in Sec. IV] this is not always the case. However, it is expected that $\partial \hat{C}$ is sufficiently well-behaved so that Eq. (1.2) holds over a sufficiently large subset of $\partial \hat{C}$ that $\hat{C}$ is indeed a future trapped region, in the sense that it must lie in the black-hole region of the spacetime. In particular, we conjecture that $\partial \hat{C}$ is everywhere $C^{2-}$ (see below) and $C^{2}$ everywhere except on a closed set $\mathcal{Z}$ of measure zero. (See Sec. IV for the statement and discussion of this conjecture.) Therefore, although Eq. (1.1) may not hold everywhere on $\partial \hat{C}$, it does hold on $\partial \hat{C} \backslash \mathcal{Z}$, which, with the fact that the surface is $C^{2-}$, is sufficient to guarantee the the region is trapped. (See theorem 5 in Sec. VB.) This proves the following strengthened version of Eardley's theorem.

Theorem 1. Fix an initial data set for the gravitational field $\left(\Sigma, g_{a b}, K_{a b}\right)$ [1] and fix a subset $\Omega \subset \Sigma$ that is a compact three-manifold with $C^{2}$ boundary. If $K_{a b} h^{a b}$ is no less than the surface-to-volume ratio of $\Omega$ for all rank 2 orthogonal projection maps $h^{a}{ }_{b}$ [15] everywhere on $\Omega$, then there exists a future trapped region in $\Omega$, provided conjecture 1 (stated in Sec. IV) holds for $\left(\Omega, g_{a b}\right)$.

Denoting the eigenvalues of $K^{a}{ }_{b}$ by $\left(k_{1}, k_{2}, k_{3}\right)$, ordered so that $k_{1} \leq k_{2} \leq k_{3}$, it is worth noting that the minimum of $K_{a b} h^{a b}$ over all rank 2 orthogonal projection maps $h^{a}{ }_{b}$ [15] is precisely $k_{1}+k_{2}$. Therefore, the sole condition of theorem 1 is that the sum of the two lesser principal (extrinsic) curvatures be no less than the surface-to-volume ratio of $\Omega$, everywhere on $\Omega$.

We can now assert (assuming conjecture 1 ) that if such a region $\Omega$ exists in a Cauchy surface of an asymptotically flat spacetime [4] satisfying the null-convergence condition [3], the spacetime must contain a black hole with the future trapped region therein.

Comparing the two theorems, we see that while neither locates the future apparent horizon, theorem 1 does tell us some subset of $\Omega$, namely $\hat{C}$, is contained within the future apparent horizon. Further, we see that theorem 1 dispenses with the asymptotic and energy conditions that were needed by Eardley because of their use in Schoen and Yau's analysis of Jang's equation.

The remainder of this work is organized as follows. In Sec. II, we prove the lemma providing the bound $H \leq$ $\sigma(\Omega)$ on $\partial \hat{C}$. In Sec. III, we review Eardley's argument and then present a strengthened version of theorem 1 . In Sec. IV, we state and discuss the two conjectures in Riemannian geometry needed for this work. In Sec. \, we offer a new proof that future trapped regions are trapped, which is then modified to establish the same result for our weaker notion a future trapped region, and then we discuss the possibility of further extending the notion of a future trapped region. Lastly, in Sec. VI, we discuss the strengths and weakness of our results.

Our conventions are those of Ref. [4] with the notable exception that our sign convention for the extrinsic cur- 
vature of our initial data surfaces is such that positive $K$ is associated with collapse in the sense that it measures the convergence of future-directed geodesic normals to the surface. On the other hand, $H$ measures the divergence of the outward geodesic normals to the surface of a region within an initial data surface.

Recall that a map between manifolds is said to be $C^{k-}$ if the the mapping is $C^{k-1}$ and its $(k-1)$-order derivatives of the functions defining the mapping are locally Lipschitz 5, 16]. Thus, a $C^{2-}$ embedded surface is $C^{1}$ and the derivative of the embedding map is locally Lipschitz.

It proves very convenient to make the following definitions. Given a manifold $N$ (possibly with boundary), define $\mathcal{C}^{k}(N)\left[\mathcal{C}^{k-}(N)\right]$ to be the collection of compact subsets of $N$ having the structure of a manifold with $C^{k}$ $\left(C^{k-}\right)$ boundary. It is useful to keep in mind that

$$
\mathcal{C}^{0}(N) \supset \mathcal{C}^{1-}(N) \supset \mathcal{C}^{1}(N) \supset \mathcal{C}^{2-}(N) \supset \mathcal{C}^{2}(N) \supset \cdots
$$

Elements of $\mathcal{C}^{k}(N)$ and $\mathcal{C}^{k-}(N)$ need not be connected, i.e., they can have many connected components. Further, if $\Omega \in \mathcal{C}^{k}(N)$, then $\Omega \in \mathcal{C}^{k}(\Omega)$, and, similarly, if $\Omega \in$ $\mathcal{C}^{k-}(N)$, then $\Omega \in \mathcal{C}^{k-}(\Omega)$.

Lastly, for a map $\phi: A \rightarrow B, \phi[A]$ denotes the image of $A$ in $B, A \backslash B$ denotes the set of elements in $A$ that are not in $B$, and $\bar{A}$ denotes the closure of $A$.

\section{PROOF THAT $H \leq \sigma(\Omega)$ ON $\partial \hat{C}$}

Denote the surface area, volume, and surface-tovolume ratio of a region $C \in \mathcal{C}^{1}(\Sigma)$ by $A(C), V(C)$, and $\sigma(C)=A(C) / V(C)$, respectively. More explicitly,

$$
\begin{aligned}
& A(C)=\int_{\partial C} \epsilon_{a b}, \\
& V(C)=\int_{C} \epsilon_{a b c},
\end{aligned}
$$

where $\epsilon_{a b c}$ is the volume element constructed from $g_{a b}$ and $\epsilon_{a b}$ is the volume element constructed from the metric $h_{a b}$ induced on $\partial C$ by $g_{a b}$.

Lemma 1. Fix a pair $\left(\Omega, g_{a b}\right)$, where $\Omega$ is a compact three-dimensional manifold with $C^{1}$ boundary and $g_{a b}$ is a smooth Riemannian metric on $\Omega$. If $\hat{C} \in \mathcal{C}^{1}(\Omega)$ is such that $\sigma(\hat{C}) \leq \sigma(C)$ for all $C \in \mathcal{C}^{1}(\Omega)$ and $O$ is an open subset of $\partial \hat{C}$ where the surface is $C^{2}$, then $H \leq \sigma(\hat{C}) \leq$ $\sigma(\Omega)$ on $O$, where $H$ is the mean extrinsic curvature of $\partial \hat{C}$ [6]. If, further, $O$ is in the interior of $\Omega$, then $H=\sigma(\hat{C})$ on $O$.

Proof. The idea of the proof is simple: We calculate $\sigma$ as a function along certain well-behaved curves in $\mathcal{C}^{1}(\Omega)$ containing $\hat{C}$, calculate its derivative at $\hat{C}$, and then use the fact that $\hat{C}$ minimizes $\sigma$ in $\mathcal{C}^{1}(\Omega)$.
Although there are many curves in $\mathcal{C}^{1}(\Omega)$, by which we mean one-parameter family of regions $C_{\lambda} \in \mathcal{C}^{1}(\Omega)$, for simplicity, we shall restrict ourselves to families arising from a smooth deformation of a region $C \in \mathcal{C}^{1}(\Omega)$ in the sense that $C_{\lambda}=\phi_{\lambda}[C]$ for some one-parameter family of maps $\phi_{\lambda}: \Omega \rightarrow \Omega$ such that $\phi_{\lambda}$ is a diffeomorphism between $\Omega$ and $\phi_{\lambda}[\Omega]$, with $\phi_{0}$ being the identity map on $\Omega$. Our requirement that $\Omega$ and $\phi_{\lambda}[\Omega]$ be diffeomorphic is sufficient to guarantee that $\partial\left(\phi_{\lambda}[C]\right)=\phi_{\lambda}[\partial C]$, which makes the following calculations easier than they would be otherwise.

A particularly simple class of such deformations, which is sufficient for our purposes, are those associated with the flows of fixed vector fields on $\Omega$ [16]. [That is, given a fixed vector field $\xi^{a}$, for $p \in \Omega, \phi_{\lambda}(p)$ is the point along the integral curve of $\xi^{a}$ containing $p$ a parameter distance $\lambda$ from $p$.] In order that these deformations be well defined on all of $\Omega$ for some positive $\lambda$, it is necessary to restrict ourselves to vector fields that are inward pointing everywhere on $\partial \Omega$ (where we consider vectors tangent to $\partial \Omega$ as inward pointing, so $\xi^{k} n_{k} \leq 0$ everywhere on $\partial \Omega$, where $n^{k}$ is the unit outward normal to $\partial \Omega$ ). Otherwise, a point $p \in \partial \Omega$ where $\xi^{a}$ is strictly outward pointing would be mapped "out of" $\Omega$, and hence the deformation constructed from it would not be defined for any positive $\lambda$, no matter how small. A deformation $\phi_{\lambda}$ constructed from an inward pointing vector field is well defined for all $\lambda \geq 0$ and is a diffeomorphism between $\Omega$ and $\phi_{\lambda}[\Omega]$.

Fix any inward pointing vector field $\xi^{a}$ whose support intersects $\partial C$ within $O$ and construct its one-parameter family of deformations $\phi_{\lambda}$. Evaluating $A$ and $V$ on $C_{\lambda}=$ $\phi_{\lambda}[C]$ using Eqs. (2.1), differentiating with respect to $\lambda$, and then evaluating at $\lambda=0$, we find that

$$
\begin{aligned}
A^{\prime}(C) & =\int_{O} H\left(\xi^{k} n_{k}\right) \epsilon_{a b}, \\
V^{\prime}(C) & =\int_{O}\left(\xi^{k} n_{k}\right) \epsilon_{a b},
\end{aligned}
$$

where $H$ is the mean extrinsic curvature of $\partial C$ and $n^{k}$ is the outward unit normal to $\partial C$. Differentiating the equality $\sigma\left(C_{\lambda}\right)=A\left(C_{\lambda}\right) / V\left(C_{\lambda}\right)$, evaluating at $\lambda=0$, and using Eqs. (2.2), we find that

$$
\sigma^{\prime}(C)=\frac{1}{V(C)} \int_{O}(H-\sigma(C))\left(\xi^{k} n_{k}\right) \epsilon_{a b}
$$

Using this equation, we now establish our bound on $H$.

We begin with the case where $O$ is in the interior of $\Omega$. Fix any point $p \in O$. To show that $H(p)=\sigma(\hat{C})$, suppose, for contradiction, that $H(p)>\sigma(\hat{C})$. Then, using the facts that $p$ is in the interior of $\Omega$ and $H$ is continuous at $p$, it is not difficult to show that there exists an open neighborhood $N$ of $p$ and a vector field $\xi^{a}$ such that: (1) the support of $\xi^{a}$ is $\bar{N}$; (2) $\partial \hat{C}$ is $C^{2}$ on $\bar{N} \cap \partial \hat{C} ;(3)(\bar{N} \cap \partial \Omega)=\emptyset ;(4)(H-\sigma(\hat{C}))>0$ on $N \cap \partial \hat{C}$; (5) $\left(\xi^{k} n_{k}\right)<0$ on $N \cap \partial \hat{C}$. Notice that $\xi^{a}$, being zero on $\partial \Omega$, is inward pointing, so the one-parameter family 
of deformations $\phi_{\lambda}$ constructed from $\xi^{a}$ is defined for all $\lambda \geq 0$. Using Eq. (2.3), we see that $\sigma^{\prime}(\hat{C})<0$, which is impossible as otherwise, for sufficiently small $\lambda$, the region $\phi_{\lambda}[\hat{C}]$ would have a smaller surface-to-volume ratio than $\hat{C}$. Similarly, if $H(p)<\sigma(\hat{C})$, there exists an open neighborhood $N$ of $p$ and a vector field $\xi^{a}$ satisfying the above except with the inequalities in (4) and (5) both reversed. Using Eq. (2.3), we again find that $\sigma^{\prime}(\hat{C})<0$, which is again a contradiction. Therefore, $H(p)=\sigma(\hat{C})$, as claimed.

Otherwise, fix any point $p \in O$. To show that $H(p) \leq$ $\sigma(\hat{C})$, suppose, for contradiction, that $H(p)>\sigma(\hat{C})$. Then, there exists an open neighborhood $N$ of $p$ and a vector field $\xi^{a}$ satisfying the above with (3) replaced by: (3') $\xi^{a}$ is inward pointing on $\bar{N} \cap \partial \Omega$. Again the oneparameter family of deformations $\phi_{\lambda}$ constructed from $\xi^{a}$ is defined for all $\lambda \geq 0$. Using Eq. (2.3), we see that $\sigma^{\prime}(\hat{C})<0$, which is contradicts the minimality of $\sigma$ at $\hat{C}$. Therefore, $H(p) \leq \sigma(\hat{C})$, as claimed.

Lastly, that $\sigma(\hat{C}) \leq \sigma(\Omega)$ follows simply from the facts that $\hat{C}$ minimizes $\sigma$ over $\mathcal{C}^{1}(\Omega)$ and $\Omega \in \mathcal{C}^{1}(\Omega)$. This completes the proof of lemma 1 .

\section{STRENGTHENING THEOREM 1}

We begin with two definitions. First define the scalar field $\kappa$ on $\Sigma$ by setting

$$
\kappa(p)=\min _{h^{a}{ }_{b}}\left(K_{a b} h^{a b}\right),
$$

for each $p \in \Sigma$, where the minimum is over the set of all rank 2 orthogonal projection maps $h^{a}{ }_{b}$ at $p$ 15. That is, $\kappa(p)$ is the sum of the two lesser principal (extrinsic) curvatures at $p$. Second, for any continuous function $f$ on $\Sigma$, define the function $W_{f}$ on $\mathcal{C}^{1}(\Sigma)$ by setting

$$
W_{f}(C)=\int_{C} f \epsilon_{a b c},
$$

for each $C \in \mathcal{C}^{1}(\Sigma)$. Note that with $f=1, W_{1}(C)=$ $V(C)$.

The idea behind the proof of theorem 1 was to use the properties of the region that minimizes the surface-tovolume ratio $\sigma$ on $\mathcal{C}^{1}(\Omega)$. Noting that $\sigma=A / V=A / W_{1}$, one way to proceed in generalizing theorem 1 is to analyze the properties of the region that minimizes $A / W_{\kappa}$ on $\mathcal{C}^{1}(\Omega)$. Assuming the relevant generalized version of conjecture 1 holds, it can be shown that there is a future trapped region in $\Omega$ provided that $A(\Omega) / W_{\kappa}(\Omega) \leq 1$ and $\kappa$ is non-negative (and not everywhere zero) on $\Omega$. However, such an argument must fail if $\kappa$ is negative somewhere on $\Omega$ since, by choosing regions with large area in regions where $\kappa$ is negative, we can find $C \in \mathcal{C}^{1}(\Sigma)$ for which the ratio $A(C) / W_{\kappa}(C)$ is negative and as large as we wish (i.e., $A / W_{\kappa}$ has no finite lower bound in this case). The fact that $\kappa$ cannot be even slightly negative on small subsets of $\Omega$ makes this route unattractive, so we take an alternative path suggested by the argument Eardley used in proving his theorem.

\section{A. Eardley's argument}

Fix an asymptotically flat initial data set for the gravitational field $\left(\Sigma, g_{a b}, K_{a b}\right)$ [1] with sources satisfying the dominant energy condition. Schoen and Yau have shown that such an initial data set does not contain an apparent horizon (either future or past) if and only if there exists a scalar field $f$ satisfying Jang's equation everywhere on $\Sigma$ [12]. Eardley's argument is that certain initial data are inheritly incompatible with the existence of a global solution of Jang's equation, and therefore a (future or past) apparent horizon must be present. This argument goes as follows.

Defining

$$
h^{a}=\frac{D^{a} f}{\sqrt{1+D^{m} f D_{m} f}},
$$

where $D_{a}$ is the derivative operator on $\Sigma$ associated with $g_{a b}$, Jang's equation takes the simple form

$$
D_{a} h^{a}=K_{a b}\left(g^{a b}-h^{a} h^{b}\right) .
$$

Noting that $h^{m} h_{m}<1$ everywhere, define the scalar field $\tilde{\kappa}$ on $\Sigma$ by setting

$$
\tilde{\kappa}(p)=\inf _{|x|<1}\left(K_{a b}\left(g^{a b}-x^{a} x^{b}\right)\right),
$$

at each point $p \in \Sigma$, where the infimum is over all vectors $x^{a}$ at $p$ with $x^{m} x_{m}<1$. (By continuity, the value of $\tilde{\kappa}$ is unchanged if we modify its definition by taking the minimum over all vectors $x^{a}$ at $p$ with $x^{m} x_{m} \leq 1$.) Using the fact that for $x^{a} \neq 0$

$$
\begin{aligned}
K_{a b}\left(g^{a b}-\right. & \left.x^{a} x^{b}\right) \\
= & \left(1-x^{m} x_{m}\right) K_{a b} g^{a b} \\
& +\left(x^{m} x_{m}\right) K_{a b}\left(g^{a b}-x^{a} x^{b} /\left(x^{m} x_{m}\right)\right),
\end{aligned}
$$

it is not difficult that show that

$$
\tilde{\kappa}=\min \left(K^{a}{ }_{a}, \kappa\right) \leq \kappa
$$

at each point. Define the function $\tilde{S}$ on $\mathcal{C}^{1}(\Sigma)$ by setting

$$
\tilde{S}(C)=A(C)-W_{\tilde{\kappa}}(C),
$$

for each $C \in \mathcal{C}^{1}(\Sigma)$.

If a global solution of Jang's equation exists, it follows that

$$
D_{a} h^{a} \geq \tilde{\kappa},
$$

everywhere on $\Sigma$. Integrating Eq. (3.9) over any region $C \in \mathcal{C}^{1}(\Sigma)$ and using the fact that $h^{\kappa} n_{k}<1$ everywhere 
on $\partial C$, where $n^{k}$ is the outward unit normal to $\partial C$, we find that

$$
\tilde{S}(C)>0
$$

That is, $\tilde{S}$ is a strictly positive function on $\mathcal{C}^{1}(\Sigma)$. Therefore, if there exists a region $\Omega \in \mathcal{C}^{1}(\Sigma)$ with $\tilde{S}(\Omega) \leq 0$, a global solution of Jang's equation cannot exist, and, thus, by Schoen and Yau's results, a (future or past) apparent horizon must be present within $\Sigma$.

In this argument, we see that the function $\tilde{S}$ on $\mathcal{C}^{1}(\Sigma)$ arises rather naturally. This suggests that we should attempt to strengthen the above result by showing that when there exists $\Omega \in \mathcal{C}^{1}(\Sigma)$ with $\tilde{S}(\Omega) \leq 0$ a future trapped region must exist within $\Omega$ (without the need for any asymptotic or stress-energy conditions). However, it turns out that we can do a little better using the function $S$ on $\mathcal{C}^{1}(\Sigma)$, defined by

$$
S(C)=A(C)-W_{\kappa}(C),
$$

for each $C \in \mathcal{C}^{1}(\Sigma)$, rather than $\tilde{S}$. Since $\tilde{\kappa} \leq \kappa$, it follows that $S(C) \leq \tilde{S}(C)$, and, hence, if $\tilde{S}(\Omega) \leq 0$, then $S(\Omega) \leq 0$. So a future trapped region theorem using $\tilde{S}$ follows from such a theorem for $S$. (See theorem 3, below).

It is worth noting that for many initial data sets, $S$ and $\tilde{S}$ will coincide. Using the facts that $K^{a}{ }_{a}=k_{1}+k_{2}+k_{3}$ and $\kappa=k_{1}+k_{2}$, it follows that $\tilde{\kappa}<\kappa$ if and only if $K_{a b}$ is negative definite $\left(K_{a b} x^{a} x^{b}<0\right.$ for all nonzero $\left.x^{a}\right)$, and $\tilde{\kappa}=\kappa$ otherwise. Therefore, if $K_{a b}$ is nowhere negative definite on $\Sigma$, i.e., nowhere is the surface positively contracting in all directions, then $S(C)=\tilde{S}(C)$ for all $C \in \mathcal{C}^{1}(\Sigma)$.

\section{B. New argument}

Our first notable property of $S$ is that any region $\hat{C} \in$ $\mathcal{C}^{2}(\Sigma)$ that is a stationary point of $S$ is a future trapped region.

Theorem 2. If $\hat{C} \in \mathcal{C}^{2}(\Sigma)$ is a stationary point of $S$ (in the sense that $S^{\prime}(\hat{C})=0$ for all smooth variations of $C$ ), then $\hat{C}$ is a future trapped region.

Proof. Fix any region $C \in \mathcal{C}^{2}(\Sigma)$ and any open subset $O$ of $\partial C$. Then, for all smooth vector fields $\xi^{a}$ whose support intersects $\partial C$ within $O$

$$
S^{\prime}(C)=\int_{O}(H-\kappa)\left(\xi^{k} n_{k}\right) \epsilon_{a b} .
$$

Therefore, repeating the argument used in lemma 1 and using the fact that $\hat{C}$ is in the interior of $\Sigma$ (as $\Sigma$ has no boundary), we find that $H=\kappa$ on $\partial \hat{C}$. However, as $\kappa \leq K_{a b} h^{a b}$ on $\partial \hat{C}$, where $h^{a b}$ is the metric induced on $\partial \hat{C}, H \leq K_{a b} h^{a b}$ on $\partial \hat{C}$. Therefore, $\hat{C}$ is a future trapped region.
Note that if $\hat{C} \in \mathcal{C}^{2}(\Sigma)$ is a local minimum of $S$ in the sense that there is an open set $N \subset \Sigma$ such that $S(\hat{C}) \leq S(C)$ for all $C \in \mathcal{C}^{2}(\Sigma)$ with $C \subset N$, then $\hat{C}$ is a stationary point of $S$. Further, for momentarily static initial data sets $\left(K_{a b}=0\right.$ on $\left.\Sigma\right), \kappa=0$ on $\Sigma$, so $S$ is simply the surface area of $C$. Therefore, in this case, the problem of finding stationary points of $S$ is exactly the problem of finding surfaces whose area is stationary (in the sense of theorem 2), e.g., minimal two-surfaces [17].

Since finding stationary points of $S$ is a difficult task, it is desirable to have an alternate condition that guarantees the existence of a future trapped region. Mimicking the proof of theorem 1, we fix a region $\Omega \in \mathcal{C}^{2}(\Sigma)$ and then analyze the properties of a region that minimizes $S$ on $\mathcal{C}^{1}(\Omega)$. If $S(\Omega)>0, S$ may not have a minimum on $\mathcal{C}^{1}(\Omega)$. To see this, note that as there exist regions with arbitrarily small surface areas and volumes, $\inf _{\mathcal{C}^{1}(\Omega)}(S) \leq 0$. Yet, for an initial data set with $\kappa \leq 0$ (as is the case for a maximal hypersurface), there is no region $\hat{C} \in \mathcal{C}(\Omega)$ that attains the infimum (being zero) as any such region necessary has $S(\hat{C}) \geq A(\hat{C})>0$. However, for any region $\Omega$ with $S(\Omega) \leq 0$, we conjecture that $S$ does have a minimum on $\mathcal{C}^{1}(\Omega)$. (In fact, we conjecture that the minimizing region $\hat{C}$ is a member of $\mathcal{C}^{2-}(\Omega)$ and has further nice differentiable properties. See conjecture 2 in Sec. IV.) The idea behind this conjecture is that if $\inf _{\mathcal{C}^{1}(\Omega)}(S)<0$ (which is guaranteed to be the case if $S(\Omega)<0)$, a sequence of regions $C_{i}$ with $S\left(C_{i}\right)$ approaching this infimum cannot become degenerate in the sense that their volumes go to zero or their areas become infinite, while if $\inf _{\mathcal{C}^{1}(\Omega)}(S)=0$, then $S(\Omega)=0$, so $\Omega$ itself is a minimizing region. Note that $\inf _{\mathcal{C}^{1}(\Omega)}(S)$ must be finite as

$$
\inf _{\mathcal{C}^{1}(\Omega)}(S) \geq-\max _{\Omega}(\kappa) V(\Omega)
$$

a lower bound that holds even if $\kappa$ is negative somewhere on $\Omega$. This is to be compared to the difficulty in establishing a similar result for the surface-to-volume ratio function $\sigma$ and lack of any finite lower bound on $A / W_{\kappa}$ when $\kappa$ is negative somewhere on $\Omega$. Using these ideas, the following theorem shows that if $S$ is not strictly positive on $\mathcal{C}^{2}(\Sigma)$, then $\Sigma$ must contain a future trapped region.

Theorem 3. If $S(\Omega) \leq 0$ for some $\Omega \in \mathcal{C}^{2}(\Sigma)$, then there exists a future trapped region in $\Omega$, provided conjecture 2 (stated in Sec. IV) holds for $\left(\Omega, g_{a b}\right)$.

Proof. By conjecture 2, there exists $\hat{C} \in \mathcal{C}^{2-}(\Omega)$ that minimizes $S$ on $\mathcal{C}^{1}(\Omega)$ and further $\partial \hat{C}$ is $C^{2}$ on $\partial \hat{C} \backslash \mathcal{Z}$, where $\mathcal{Z}$ is a closed set of measure zero. Therefore, for all one-parameter family of deformations constructed from an inward pointing vector field on $\Omega$ whose support intersects $\partial \hat{C}$ where the surface is $C^{2}$, we have $0 \leq S^{\prime}(\hat{C})$. Using Eq. (3.12), with $C=\hat{C}$ and repeating the argument used in lemma 1 , we find that $H \leq \kappa$ on $\partial \hat{C} \backslash \mathcal{Z}$. However, as $\kappa \leq K_{a b} h^{a b}$ on all of $\partial \hat{C}$, where $h^{a b}$ is the 
metric induced on $\partial \hat{C}, H \leq K_{a b} h^{a b}$ on $\partial \hat{C} \backslash \mathcal{Z}$. Therefore, $\hat{C}$ is a future trapped region.

Note that if $\kappa \leq 0$ on $\Sigma$ (as is the case for maximal hypersurfaces), then there is no region $\Omega$ meeting the condition of theorem 3 as $S(\Omega) \geq A(\Omega)>0$. Further, the condition of Eardley's theorem and theorem 1 that $\sigma(\Omega) \leq \min _{\Omega}(\kappa)$ implies that $A(\Omega) \leq \min _{\Omega}(\kappa) V(\Omega) \leq$ $W_{\kappa}(\Omega)$ and, therefore, $S(\Omega) \leq 0$, which is the sole condition of theorem 3 . Therefore, theorem 3 is stronger than theorem 1, which is stronger than Eardley's theorem.

It is interesting to note that $S(C)$ can be expressed as a pure surface integral by introducing any vector field $\zeta^{a}$ on $\Sigma$ (or merely on $\Omega$ ) having the property that $D_{a} \zeta^{a}=\kappa$, where $D_{a}$ is the derivative operator associated with the metric $g_{a b}$. With this, we have

$$
S(C)=\int_{\partial C}\left(1-\zeta^{k} n_{k}\right) \epsilon_{a b}
$$

For instance, a particularly simple choice of $\zeta^{a}$ is that given by taking $\zeta^{a}=D^{a} \phi$ for a scalar field $\phi$. Then, $\phi$ must be a solution of Poisson's equation $D_{a} D^{a} \phi=\kappa$ and can be fixed uniquely by fixing boundary data for $\phi$ on $\partial \Omega$ (e.g., $\phi=0$ on $\partial \Omega$ ) or a boundary condition on $\phi$ at infinity (though whether this can always be accomplished is more subtle). We will not pursue this formulation any further here as nothing new seems to gained from this viewpoint.

In theorems 1 and 3, we have restricted ourselves regions $\Omega$ with $C^{2}$ boundary for the sake of simplicity, and we expect that both theorems hold under weaker conditions. It would seem that the weakest differentiability condition that should be imposed is that for which it makes sense for a region to speak of a region being future trapped.

\section{TWO GEOMETRICAL CONJECTURES}

The relevance of theorems 1 and 3 rests heavily upon the following two conjectures, which we believe to be true.

Conjecture 1. Fix a pair $\left(\Omega, g_{a b}\right)$, where $\Omega$ is a compact three-dimensional manifold with $C^{2}$ boundary and $g_{a b}$ is a smooth Riemannian metric on $\Omega$. There exists $\hat{C} \in \mathcal{C}^{2-}(\Omega)$ such that $\sigma(\hat{C}) \leq \sigma(C)$ for all $C \in \mathcal{C}^{1}(\Omega)$. [In other words, $\sigma$ has a minimum on $\mathcal{C}^{1}(\Omega)$ and a minimizing region is a member of $\mathcal{C}^{2-}(\Omega)$.] Further, $\partial \hat{C}$ is $C^{2}$ everywhere except on the closed set of measure zero given by $\partial \mathcal{W}$, where $\mathcal{W}=(\partial \Omega \cap \partial \hat{C})$ (and $\partial \mathcal{W}$ is constructed viewing $\mathcal{W}$ as a subset of either $\partial \Omega$ or $\partial \hat{C})$.

Conjecture 2. Fix a triple $\left(\Omega, g_{a b}, \kappa\right)$, where $\Omega$ is a compact three-dimensional manifold with $C^{2}$ boundary, $g_{a b}$ is a smooth Riemannian metric on $\Omega$, and $\kappa$ is a smooth scalar field on $\Omega$. If $S(\Omega) \leq 0$, then there exists $\hat{C} \in \mathcal{C}^{2-}(\Omega)$ such that $S(\hat{C}) \leq S(C)$ for all $C \in \mathcal{C}^{1}(\Omega)$. [In other words, $S$ has a minimum on $\mathcal{C}^{1}(\Omega)$ and a minimizing region is a member of $\mathcal{C}^{2-}(\Omega)$.] Further, $\partial \hat{C}$ is $C^{2}$ everywhere except on the closed set of measure zero given by $\partial \mathcal{W}$, where $\mathcal{W}=(\partial \Omega \cap \partial \hat{C}$ ) (and $\partial \mathcal{W}$ is constructed viewing $\mathcal{W}$ as a subset of either $\partial \Omega$ or $\partial \hat{C})$.

Note that although $\partial \mathcal{W}$ is by its definition a closed subset of $\partial \hat{C}$, its being a set of measure does not appear to be guaranteed as there exist boundaries of positive measure.

In conjectures 1 and 2, we have asserted that the surface $\partial \hat{C}$ is a $C^{2-}$ submanifold that is almost everywhere $C^{2}$. It is too much to expect that $\partial \hat{C}$ will be everywhere $C^{2}$ as we expect a discontinuity in its mean extrinsic curvature $H$ where $\partial \hat{C}$ "first intersects" $\partial \Omega$, i.e., on $\partial \mathcal{W}$. To see this, suppose conjectures 1 and 2 are true. Then, write $\partial \hat{C}$ as the disjoint union of three sets as follows

$$
\partial \hat{C}=(\partial \hat{C} \backslash \mathcal{W}) \cup(\mathcal{W} \backslash \partial \mathcal{W}) \cup(\partial \mathcal{W}) .
$$

As $\partial \hat{C} \backslash \mathcal{W}$ is in the interior of $\Omega, H=\sigma(\hat{C})$ and $H=\kappa$ in conjectures 1 and 2, respectively. However, as $\partial \hat{C}$ coincides with $\partial \Omega$ on the open set $\mathcal{W} \backslash \partial \mathcal{W}, H$ will equal the mean extrinsic curvature of $\partial \Omega$ on $\mathcal{W} \backslash \partial \mathcal{W}$. Therefore, in general, we expect that $H$ will suffer a discontinuity on $\partial \mathcal{W}$. So, as $H$ will not always be $C^{2}, \partial \hat{C}$ will not always be $C^{2}$. However, note that this argument suggests that the lack in continuity in the second-order partial derivatives defining the surface arise from mere jumps and not divergences. It is this property that suggests that the surface is $C^{2-}$.

While we shall not attempt to do so here, conjectures 1 and 2 can probably be proven using the ideas and techniques of geometric measure theory [18]. Very roughly, we consider a subset $\mathcal{V}(\Omega)$ of $\mathcal{C}^{1-}(\Omega)$ whose members are sufficiently well-behaved that they have finite volume and surface area (using the Hausdorff measure). One then argues that $S$ is a continuous function (in some natural topology) on $\mathcal{V}(\Omega)$ and that the subset of $\mathcal{V}(\Omega)$ defined by those $C \in \mathcal{V}(\Omega)$ such that $S(C) \leq S(\Omega)$ is compact. It then follows immediately that there is a region $\hat{C} \in \mathcal{V}(\Omega)$ that achieves the minimal value of $S$ on this set. The last step would be to establish that $\hat{C}$ is actually a member of $\mathcal{C}^{2-}(\Omega)$ and $C^{2}$ on $\partial \hat{C} \backslash \mathcal{W}$ (and that $\mathcal{W}$ is a set of measure zero). We leave the task of showing that these steps can actually be completed open for investigation.

\section{FUTURE TRAPPED REGIONS ARE TRAPPED}

Although there exists theorems showing that future trapped regions must lie within the black hole region of the spacetime, the arguments, as given, require that their surfaces be everywhere $C^{2}$ 回, 5]. Here, we show that the same result holds for regions with boundaries that are not quite this smooth, and so deserve to be called future trapped regions. To make our method of proof clear, we first cover the case where the surface of the region is everywhere $C^{2}$. After this, we modify the proof to 
accommodate our more general regions. We then discuss the possibility of further generalizations.

While our method of proof is similar to the existing proofs for smooth regions, there is a notable difference in the final derived contradiction. The Hawking and Ellis argument ends with the contradiction that the area of $\partial C$ is no less than the area of $\partial J^{+}(C) \cap \mathcal{J}^{+}$, which, being at infinity, is infinite. The Wald argument ends with the contradiction that the future expansion of the null generators of $\partial J^{+}(C)$ is nonpositive on $\partial C$ and yet positive near $\mathcal{J}^{+}$. Here, we end with the contradiction that there are null generators of $\partial J^{+}(C)$ extending beyond $\mathcal{J}^{+}$that possess a point conjugate to $\partial C$ on $\mathcal{J}^{+}$.

Actually, it should be noted that the Wald argument contains a slight error in that the local cross-sections of $\mathcal{J}^{+}$constructed need not have the requisite differentiability properties in order that nearby cross-sections of $\partial J^{+}(C)$ have strictly positive future expansion. A simple counterexample is provided by a smooth closed region $C$ in a flat spatial hypersurface $\Sigma$ in Minkowski spacetime with the property that all of $C$ lies to one side of a flat plane $\mathcal{P}$ in $\Sigma$ except for a closed region $\partial C \cap \mathcal{P}$ having a non-empty interior (as a subset of $\mathcal{P}$ ). Then, it is not difficult to see that the null generators of $\partial J^{+}(C)$ having past endpoint on $\partial C \cap \mathcal{P}$ intersect $\mathcal{J}^{+}$and have zero expansion everywhere. Of course, the Wald argument can easily be fixed by introducing an area type argument, or by adopting the method of theorem 4, which can be viewed as such a fix as it has much of its inspiration from the Wald argument.

Our notion of asymptotic flatness is that given in Ref. [4. We denote the manifolds of the "physical" and "unphysical" spacetime by $M$ and $M^{\prime}$, respectively. We remind the reader that $M=M^{\prime} \backslash\left(\overline{J^{+}\left(i^{0}\right)} \cup \overline{J^{-}\left(i^{0}\right)}\right)$, where $i^{0}$ is the point representing spatial infinity. Therefore, $\partial M=\left(i^{0} \cup \mathcal{J}^{+} \cup \mathcal{J}^{-}\right)$, where $\mathcal{J}^{ \pm}=\left(\partial J^{ \pm}\left(i^{0}\right)\right) \backslash i^{0}$ are future and past null infinity.

Furthermore, the theorems we prove are for strongly asymptotically predictable spacetimes [4], which are simply those asymptotically flat spacetimes for which there exists an open globally hyperbolic subset $V$ of $M^{\prime}$ containing $\overline{J^{-}\left(\mathcal{J}^{+}\right) \cap M}$ (where the closure is as a subset of $M^{\prime}$ ). Note that $\partial M \subset V$. It can be shown that all globally hyperbolic asymptotically flat spacetimes are strongly asymptotically predictable. Further, the globally hyperbolic asymptotic region $V$ can be chosen so that it contains all of $M$ and an asymptotically flat Cauchy surface $\Sigma$ for $M$ together with spatial infinity $i^{0}$ is a Cauchy surface for $V$. Therefore, the requirement that a subset $C$ of $\Sigma$ be closed and bounded away from infinity (so there exists a neighborhood of $i^{0}$ disjoint from $C$ ) is equivalent to the condition that $C$ be closed as a subset of $\Sigma^{\prime}=\left(\Sigma \cup i^{0}\right)$.

\section{A. Regions whose surfaces are $C^{2}$}

Theorem 4. Fix a smooth strongly asymptotically predictable spacetime [-1] satisfying the null-convergence condition [3]. Let $\Sigma^{\prime}$ be a smooth asymptotically flat Cauchy surface for $V$ and let $C \subset\left(\Sigma^{\prime} \cap M\right)$ be a future trapped region in the sense that $C$ is a closed subset of $\Sigma^{\prime}, \partial C$ is $C^{2}$, and the convergence of the outward futuredirected null normals to $\partial C$ is everywhere non-negative. Then, $\left(C \cap J^{-}\left(\mathcal{J}^{+}\right)\right)=\emptyset$. [That is, $C \subset\left(\Sigma^{\prime} \cap B\right)$, where $B$ is the black-hole region of the spacetime.]

Proof. In the following, all of our constructions are carried out solely within the asymptotic globally hyperbolic region $V$. Therefore, statements regarding the openness or closedness of sets refer to these properties in $V$ alone. Since $C$ does not contain $i^{0}$ (as $C$ is a subset of $M$ ), $J^{+}(C)$ does not contain $i^{0}$. Further, $J^{+}(C)$ is closed, since $C$ is a closed subset of $\Sigma^{\prime}$. (See exercise 8 from chapter 8 of Ref. [4].) Therefore, there is a neighborhood of $i^{0}$ disjoint from $J^{+}(C)$.

Suppose, for contradiction, that $\left(C \cap J^{-}\left(\mathcal{J}^{+}\right)\right) \neq \emptyset$. Then, $\left(J^{+}(C) \cap \mathcal{J}^{+}\right) \neq \emptyset$, and, hence, $\left(J^{+}(C) \cap I^{+}\left(i^{0}\right)\right) \neq$ $\emptyset$. It then follows that $\left(\partial J^{+}(C) \cap I^{+}\left(i^{0}\right)\right) \neq \emptyset$. To see this, fix any point $p \in\left(J^{+}(C) \cap I^{+}\left(i^{0}\right)\right)$. Then, as there exists a timelike curve $\gamma$ from $i^{0}$ to $p$ [which must lie entirely within $I^{+}\left(i^{0}\right)$ ] and there exists an open neighborhood of $i^{0}$ disjoint from the closed set $J^{+}(C)$, the curve $\gamma$ must leave $J^{+}(C)$ and therefore intersect $\partial J^{+}(C)$, showing that $\left(\partial J^{+}(C) \cap I^{+}\left(i^{0}\right)\right) \neq \emptyset$.

Recall that if $p$ is any point on a null generator of $\partial J^{+}(C)$ whose past endpoint on $\partial C$ has an open neighborhood on which $\partial C$ is $C^{2}$, there must not be a point conjugate to $\partial C$ between $\partial C$ and $p$ [4, 5]. Pick a point $p \in\left(\partial J^{+}(C) \cap I^{+}\left(i^{0}\right)\right)$ and a null generator $\nu$ of $\partial J^{+}(C)$ containing $p$. Then $\nu$ cannot possess a point conjugate to $\partial C$ in $M$ (with respect to either the physical or unphysical metric) nor on $\mathcal{J}^{+}$(with respect to the unphysical metric). However, in the physical portion of the spacetime $M$, it follows from the null Raychaudhuri equation and the null-convergence condition that the (physical) future convergence of the null generators of $\partial J^{+}(C)$ is not only non-negative on $\partial C$, it is non-negative everywhere to the future [4.5]. Furthermore, if such a generator has positive convergence $\rho_{0}>0$ at some point, then it must possess a conjugate point within an affine parameter time $2 / \rho_{0}$ thereafter, provided the generator can be extended this far. Therefore, as $\nu$ is future complete in the physical metric in $M$ (as it intersects $\mathcal{J}^{+}$), the (physical) convergence along $\nu$ must be zero in $M$. Therefore, in the infinitesimal sense, the physical area of a bundle of outgoing future-directed null rays orthogonal to $\partial C$ is constant along $\nu$ (in $M$ ). In terms of the unphysical metric, this area is that given by the physical area multiplied by the square of the conformal factor. As this conformal factor is zero on $\mathcal{J}^{+}$, it follows that $\nu$ possesses a point conjugate to $\partial C$ where it intersects $\mathcal{J}^{+}$(with respect to the unphysical metric), which is a contradiction. 


\section{B. Regions whose surfaces are not quite $C^{2}$}

The problem with the proof of theorem 4 when $\partial C$ is not everywhere $C^{2}$ is that it may happen that because of our choice of $p$ in the last paragraph, $\nu$ may have its past endpoint at a place on $\partial C$ where the surface is not $C^{2}$, thus making the final conjugate point argument inapplicable. When $\partial C$ is everywhere $C^{2-}$ and $C^{2}$ on $\partial C \backslash \mathcal{Z}$, where $\mathcal{Z}$ is a closed set of measure zero, although we do not have complete freedom in what choice to make for $p$, it turns out we can always find one so that the past endpoint of its associated null generator has a neighborhood within $\partial C$ on which the surface is $C^{2}$, i.e., its past endpoint is somewhere on $\partial C \backslash \mathcal{Z}$. The idea is that it is impossible for only the generators of $\partial J^{+}(C)$ with past endpoint on $\mathcal{Z}$ to make it beyond $\mathcal{J}^{+}$as there are not "enough of them" to make up a "local piece" of $\partial J^{+}(C)$, as $\mathcal{Z}$ is a set of measure zero in $\partial C$.

We capture this idea using the notion of Hausdorff measure [18]. On a differentiable manifold $N$ with Riemannian metric, for any two points $a$ and $b$ in $N$, define $d(a, b)$ to be the greatest lower bound on the lengths of $C^{1}$ curves in $N$ connecting $a$ to $b$ [so $(N, d)$ is a metric space]. For any subset $S \subset N$, set $\operatorname{diam}(S)=\sup _{a, b \in S}(d(a, b))$. Then, for any subset $A \subset N$ and numbers $k$ and $\delta>0$, set

$$
\mathcal{H}_{\delta}^{k}(A)=\inf \sum_{j} \nu_{k}\left(\frac{\operatorname{diam}\left(S_{j}\right)}{2}\right)^{k}
$$

where $\nu_{k}$ is the volume of a unit-ball in flat $\mathbb{R}^{k}$ when $k$ is a non-negative integer ( $\operatorname{so} \nu_{0}=1, \nu_{1}=2, \nu_{2}=\pi, \nu_{3}=$ $4 \pi / 3$, etc.) and an arbitrary positive constant otherwise, and where the infimum is taken over over all countable coverings $\left\{S_{j}\right\}$ of $A$ (i.e., $\left.A \subset \cup_{j} S_{j}\right)$ with $\operatorname{diam}\left(S_{j}\right) \leq \delta$. With this, the $\mathcal{H}^{k}$-measure of a set $A$ is defined as

$$
\mathcal{H}^{k}(A)=\lim _{\delta \rightarrow 0} \mathcal{H}_{\delta}^{k}(A) .
$$

This limit is well defined (though possibly infinite) as $\mathcal{H}_{\delta}^{k}(A)$ is non-decreasing in $\delta$. It is worth noting that if $\mathcal{H}^{k}(A)<\infty$ then $\mathcal{H}^{m}(A)=0$ for all $m>k$. It can be shown that if $A$ is an $k$-dimensional $C^{1}$ embedded submanifold of $N$ with $k \leq \operatorname{dim}(N)$, then $\mathcal{H}^{k}(A)$ corresponds to the usual "volume" of this submanifold. For instance, in the case $\operatorname{dim}(N)=3, \mathcal{H}^{1}(A)$ is the length of a 1-dimensional submanifold $A, \mathcal{H}^{2}(A)$ is the area of a 2-dimensional submanifold $A$, and $\mathcal{H}^{3}(A)$ is the volume of a 3-dimensional submanifold $A$.

With this, we say a subset $A$ of a differentiable manifold $N$ has $\mathcal{H}^{k}$-measure zero if $\mathcal{H}^{k}(A)=0$. It can be shown that this notion is independent of which Riemannian metric is chosen, and, therefore, whether a subset of a (paracompact) manifold has $\mathcal{H}^{k}$-measure zero is dependent solely upon the set. In the case where $k=\operatorname{dim}(N)$, $\mathcal{H}^{k}$-measure zero is identical to the usual Lebesgue notion of measure zero on a differential manifold. Furthermore, if $f$ is a locally Lipschitz map from the manifold
$N$ to another differentiable manifold, it follows that if $\mathcal{H}^{k}(A)=0$, then $\mathcal{H}^{k}(f[A])=0$.

Using these concepts, we can now prove that our generalized future trapped regions are indeed trapped.

Theorem 5. Fix a smooth strongly asymptotically predictable spacetime [4] satisfying the null-convergence condition [3]. Let $\Sigma^{\prime}$ be a smooth asymptotically flat Cauchy surface for $V$ and let $C \subset\left(\Sigma^{\prime} \cap M\right)$ be a future trapped region in the sense that $C$ is a closed subset of $\Sigma^{\prime}, \partial C$ is everywhere $C^{2-}$ and, on $\partial C \backslash \mathcal{Z}, \partial C$ is $C^{2}$ and the convergence of the outward future-directed null normals to $\partial C$ is non-negative, where $\mathcal{Z}$ is a closed set of measure zero. Then, $\left(C \cap J^{-}\left(\mathcal{J}^{+}\right)\right)=\emptyset$. [That is, $C \subset\left(\Sigma^{\prime} \cap B\right)$, where $B$ is the black-hole region of the spacetime.]

Proof. Suppose, for contradiction, that $(C \cap$ $\left.J^{-}\left(\mathcal{J}^{+}\right)\right) \neq \emptyset$. Then, using the same argument as in theorem 4 , it again follows that $\left(\partial J^{+}(C) \cap I^{+}\left(i^{0}\right)\right) \neq \emptyset$. We claim that there exists $p \in\left(\partial J^{+}(C) \cap I^{+}\left(i^{0}\right)\right)$ with an associated null generator $\nu$ having past endpoint on $\partial C \backslash \mathcal{Z}$, an open subset of $\partial C$ where the surface is $C^{2}$. We show this by arguing that there are not enough generators with past endpoint on $\mathcal{Z}$ to make up $\partial J^{+}(C)$ in $I^{+}\left(i^{0}\right)$ as follows.

First, the subset $\tilde{\mathcal{Z}}$ of $\partial J^{+}(C)$ consisting of those points with null generators having past endpoint on $\mathcal{Z}$ has $\mathcal{H}^{3}$-measure zero. To see this, denote by $\mathcal{K}$ the subset of $T V$ (the tangent bundle associated with $V$ ) consisting of all pairs $\left(p, k^{a}\right)$ where $p \in \partial C$ and $k^{a}$ is an outward future-directed null vector normal to $\partial C$ at $p$. Using the fact that $\partial C$ is $C^{2-}$, it follows that there exists a locally Lipschitz map from $\partial C \times \mathbb{R}$ onto $\mathcal{K} \subset T V$. Next, since $\partial J^{+}(C) \backslash C$ is generated by null geodesics with past endpoint on $\partial C$ and future-directed outgoing tangent vector normal to $\partial C$, we see that $\partial J^{+}(C) \backslash C$ is a subset of the projection of $\exp (\mathcal{K})$ onto $V$ (where exp is the smooth diffeomorphism from $T V$ to $T V$ defined by the geodesic flow on $T V)$. As both exp and the projection map are smooth, it follows that $\partial J^{+}(C) \backslash C$ is a subset of the image of a subset of $\partial C \times \mathbb{R}$ under a locally Lipschitz map. Therefore, since $\mathcal{Z} \times \mathbb{R}$ has $\mathcal{H}^{3}$-measure zero as a subset of $\partial C \times \mathbb{R}$ (which follows from the fact that $\mathcal{Z}$ has $\mathcal{H}^{2}$ measure zero as a subset of $\partial C$ ) and since $\tilde{\mathcal{Z}}$ is a subset of the image of a subset of $\mathcal{Z} \times \mathbb{R}$ under a locally Lipschitz map, it follows that $\tilde{\mathcal{Z}}$ has $\mathcal{H}^{3}$-measure zero in $V$. (Note that it is in the establishment of this result that we use the fact $\partial C$ is $C^{2-}$ and not merely $C^{1}$.)

Next, pick any point $q \in\left(\partial J^{+}(C) \cap I^{+}\left(i^{0}\right)\right)$ and an open neighborhood $O$ of $q$ with $O \subset I^{+}\left(i^{0}\right)$. Using the fact that $\partial J^{+}(C)$ is an achronal $C^{1-}$ embedded threedimensional submanifold of $V$ (see proposition 6.3 .1 of Ref. [5]), it follows that $\partial J^{+}(C) \cap O$ has positive $\mathcal{H}^{3}$ measure. (To see this, note that we can choose $O$ so that it is diffeomorphic to an open subset of $\mathbb{R}^{4}$ with $\partial J^{+}(C) \cap O$ corresponding to the graph of a $C^{1-}$ function of three variables.) Therefore, as the subset of $\partial J^{+}(C)$ consisting of generators with past endpoint on $\mathcal{Z}$ has $\mathcal{H}^{3}$ - 
measure zero, it follows that there must exist a point $p \in \partial J^{+}(C) \cap O$ with an associated null generator $\nu$ that has past endpoint on $\partial C \backslash \mathcal{Z}$. (In fact, there are many such points.)

Arguing as we did in theorem 4 shows that $\nu$ contains a point conjugate to $\partial C$ (with respect to the unphysical metric) where $\nu$ intersects $\mathcal{J}^{+}$(being between $\partial C$ and $p)$, which is a contradiction.

\section{Possible generalizations}

In extending the notion of a future trapped region, we have restricted ourselves to regions $C$ with $C^{2-}$ surfaces that are further $C^{2}$ everywhere except on a closed set of measure zero. We have done this because this is both what we expect of the surfaces constructed (conjectures 1 and 2) and these are regions for which we can carry through all the relevant arguments (theorems 3 and 5). However, a much greater extension seems possible. For instance, it is plausible that the notion of a future trapped region can be extended to regions with surfaces that are merely $C^{2-}$. Such a surface is twice differentiable everywhere except on a set of measure zero $\mathcal{Z}$. If the convergence of a family of future-directed outgoing null geodesics orthogonal to a surface can be defined on $\partial C \backslash \mathcal{Z}$ and the conjugate point argument used in theorem 5 can be applied to the generators with past endpoint on $\partial C \backslash \mathcal{Z}$, the notion of a future trapped region with a $C^{2-}$ surface would be a well-defined concept.

However, it would seem that the best notion of a region being future trapped would not involve any differentiability conditions. For example, consider the analogous problem of what we mean by a closed region $C$ in flat space having a surface $S$ that is everywhere locally convex. Here, we have a precise notion that imposes no differentiability conditions on the surface: For each point $p \in S$ there is a neighborhood $N$ in $S$ such that $(1-\lambda) x+\lambda y \in C$ for all $x, y \in N$ and $\lambda \in[0,1]$. (That is, the convex hull of $N$ is a subset of $C$.) Likewise, we say the surface of a region $C$ is locally concave if it is locally convex when viewed as the surface of the closure of the complement of $C$. Note that this flat space notion has a natural generalization to curved spaces: We call the surface $S$ of a closed region $C$ locally convex if for each point $p \in S$ there is a neighborhood $N$ in $S$ and a convex normal neighborhood $U$ containing $N$ such that for all points $x, y \in N$ the geodesic from $x$ to $y$ (within $U$, being unique) lies within $C$. In the $C^{2}$ case, the above implies the the extrinsic curvature $H_{a b}$ of $S$ is positive semi-definite.

We want a geometric condition that, in the $C^{2}$ case, leads to the bound $H=H^{a}{ }_{a} \leq K_{a b} h^{a b}$. Surely, such a notion would be based on a demand that the areas of all local cross sections of $\partial J^{+}(C)$ are non-increasing to the future (at least sufficiently near $\partial C$ ). The problem is to capture this idea in a well-defined sense. For instance, one needs for $\partial J^{+}(C)$ to be sufficiently well-behaved so that the surface areas of suitable cross-sections are welldefined. This is probably not such a problem as $\partial J^{+}(C)$ is an imbedded $C^{1-}$ submanifold for any set $C$. Then, to show that such regions are indeed trapped, an areatype argument similar to that used by Hawking and Ellis would probably be the most natural method to use. However, how is one to show that the areas of crosssections are non-increasing to the future when the null Raychaudhuri equation cannot be implemented? Clearly, some subtlety is needed here.

Note that a naive condition such as $\partial C$ being everywhere $C^{1-}$ and, on $\partial C \backslash \mathcal{Z}, \partial C$ is $C^{2}$ and the convergence of the outward future-directed null normals to $\partial C$ is non-negative, where $\mathcal{Z}$ is a closed set of measure zero, is insufficient. A simple counterexample is provided by taking $C$ to be a solid cube in a flat spatial hypersurface in Minkowski spacetime. Here, $\partial C$ is everywhere $C^{1-}$ and, except along the edges and vertices (a closed set $\mathcal{Z}$ of measure zero), the surface is $C^{\infty}$ and the convergence of the outward future-directed null normals to $\partial C$ is zero. However, $C$ is clearly "visible" from $\mathcal{J}^{+}$, i.e., it is not trapped. In the proof of theorem 5 , the problem with such surfaces is that one does not have a one-to-one correspondence between the null generators of $\partial J^{+}(C)$ and $\partial C$, and, as a result, the portion of $\partial J^{+}(C)$ consisting of the generators having past endpoint on $\mathcal{Z}$ has positive $\mathcal{H}^{3}$-measure. For example, at a vertex, an entire "octant's worth" of null generators of $\partial J^{+}(C)$ intersect $\partial C$ at a single point. In this case, all null generators of $\partial J^{+}(C)$ that do make it beyond $\mathcal{J}^{+}$have past endpoints on $\mathcal{Z}$.

Lastly, one might expect that a differentiability condition that would be sufficient to establish that a region $C$ is future trapped is that $\partial C$ is everywhere $C^{1}$ and $C^{2}$ on an open dense subset $D$ of $\partial C$ (with the convergence of the outward future-directed null normals being non-negative on $D$ ). In fact, this was the approach first taken herein, but was abandoned because of a difficulty. The idea is that if a null generator $\nu$ associated with a point $p \in\left(J^{+}(C) \cap I^{+}\left(i^{0}\right)\right)$ has its past endpoint on $D$, the argument proceeds as in theorem 4 , while if not, then it would seem that we could find a point arbitrarily near $p$ in $\left(J^{+}(C) \cap I^{+}\left(i^{0}\right)\right)$ with an associated generator having past endpoint on $D$. (After all, $D$ is dense in $\partial C$.) While this may be true, proving it appears to be difficult. For instance, although one might expect that there would exist a neighborhood of $\nu \cap \partial C$ (within $\partial C$ ) such that all null generators with past endpoint thereon remain on $\partial J^{+}(C)$ long enough to enter $I^{+}\left(i^{0}\right)$, it turns out that this need not be the case if we just use the fact that $\partial C$ is $C^{1}$. Whether this does hold when the additional conditions on $\partial C$ are used is not clear. 


\section{DISCUSSION}

Theorems 1 and 3 provide us with simple tests for the existence of future trapped regions within an initial data set, but how effective are they?

First, the conditions of theorems 1 and 3 are quite strong in the following sense. Recall that theorem 1 requires that $\min _{\Omega}(\kappa) \geq \sigma(\Omega)$ (as does Eardley's theorem). Using the fact that $\kappa$ is the sum of the two lesser principal (extrinsic) curvatures $\left(k_{1}, k_{2}, k_{3}\right)$, it is not difficult to show that $K^{a}{ }_{a}=k_{1}+k_{2}+k_{3} \geq \frac{3}{2} \kappa \geq \frac{3}{2} \sigma(\Omega)>0$ everywhere on $\Omega$, showing that this region is everywhere contracting "on average". However, if $K^{a}{ }_{a}$ is non-negative, $\kappa$ need not be positive. This shows that the region $\Omega$ is more than contracting "on average". Indeed, on a maximal hypersurface $\left(K^{a}{ }_{a}=0\right.$ everywhere on $\left.\Sigma\right), \kappa \leq 0$ (with equality only where $K_{a b}=0$ ) everywhere on $\Sigma$. In this respect, the condition of theorem 1 (and Eardley's theorem) is quite strong. While theorem 3 merely requires that $S(\Omega) \leq 0$, so $\kappa$ need not be positive on all of $\Omega, \kappa$ still must be positive over a sufficiently large subset of $\Omega$ in order to meet this condition.

Second, while both theorems give sufficient conditions for the existence of future trapped regions, neither condition is necessary. This is easily seen by constructing a momentarily static initial data set (so $K_{a b}=0$, and hence $\kappa=0$, on $\Sigma$ ) that contains a minimal two-surface bounding a compact region $C$. This region $C$ is future (and past) trapped and yet, as $S(C)=A(C)$ is positive, the condition of neither theorem 1 nor 3 is met.

Third, neither theorem is very sensitive to the "local" existence of a future trapped region in the following sense. Suppose we have a future trapped surface $S$ such that both families of future-directed orthogonal null congruences have strictly positive convergence on $S$. Construct a three-dimensional region $\Omega$ by "thickening" $S$ a small distance $r$ within an initial data surface containing $S$. Then, for $r$ sufficiently small, $\Omega$ is a future trapped region. However, for sufficiently small $r, \sigma(\Omega)$ will be larger than $\inf _{\Omega}(\kappa)$ and $S(\Omega)$ will be positive, and hence neither theorem enables us to deduce that $\Omega$ itself is a future trapped region.

Fourth, the conditions of theorems 1 and 3 are quite robust in the sense that if we have a region $\Omega$ that satisfies the condition of either theorem with strict inequality and then deform it to create a new region $\Omega^{\prime}$ by "pushing" very thin fingers of the surface of $\Omega$ into $\Omega$ (in arbitrarily complex ways), then, provided our fingers are sufficiently thin, the surface area, volume, and the integral of $\kappa$ for $\Omega^{\prime}$ will be sufficiently near those of $\Omega$ so that the conditions of both theorems will be met for $\Omega^{\prime}$. More generally, if we construct $\Omega^{\prime}$ by excising sufficiently thin regions from $\Omega$, both theorems guarantee the existence of a future trapped region within $\Omega^{\prime}$. This is perhaps somewhat surprising at first given that $\Omega^{\prime}$ can be topologically quite complex. However, noting that the mean curvature of the portions of the surface of $\Omega^{\prime}$ created by excising "very thin fingers" is very large and negative, we realize that $\hat{C}$ less the thin regions is nearly a future trapped region - all that is needed is a bit of adjusting near the edges where the excised region intersects $\hat{C}$.

Fifth, and last, theorems 1 and 3 do have a slight advantage in numerical search for the existence of future trapped regions as the calculation of $\sigma(\Omega)$ or $S(\Omega)$ requires only the calculation of a surface area and a volume integral, which are not as sensitive to numerical inaccuracies that would arise in calculating the mean extrinsic curvature $H$ of a two-surface in $\Sigma$ to test whether the surface is future outer trapped (i.e., testing whether the condition given by Eq. (1.1) holds on the boundary).

So, while theorems 1 and 3 do offer tests for the existence of future trapped regions, their inability to detect the existence of future trapped regions in some instances, e.g., in initial data sets associated with maximal hypersurfaces and "thin" future trapped regions, leads us to wonder whether stronger tests of the type considered here can be devised to give sufficient conditions for the existence of future trapped regions.

\section{ACKNOWLEDGMENTS}

I thank Douglas Eardley, for his encouragement in this work and for allowing me to visit the ITP at UCSB for its discussion, and Robert Geroch, for answering numerous questions.

[1] Here, $\Sigma$ is an orientable three-manifold (without boundary), representing the Universe at a given time; $g_{a b}$ is a Riemannian metric on $\Sigma$, giving the intrinsic geometry of $\Sigma$ as induced by the spacetime metric; and $K_{a b}$ is a symmetric tensor, giving the extrinsic curvature of $\Sigma$ in the spacetime.

[2] P. Anninos, et. al., Event Horizons of Numerical Black Holes, article gr-qc/9412056 on gr-qc@xxx.lanl.gov archive (to appear in the proceedings of the Seventh Marcel Grossmann Meeting on General Relativity, edited by R. Ruffini and M. Keiser, World Scientific, 1995).

[3] A spacetime with Ricci tensor $R_{a b}$ is said to satisfy the null-convergence condition if $R_{a b} k^{a} k^{b} \geq 0$ for all null $k^{a}$.

[4] R. M. Wald, General Relativity (University of Chicago Press, Chicago, 1984).

[5] S. W. Hawking and G. F. R. Ellis, The Large-Scale Structure of Space-time (Cambridge University Press, Cambridge, England, 1973).

[6] Actually, this is a slight abuse in terminology in that $H$ is the trace of extrinsic curvature of the two-surface in the three-manifold and not one-half this value. (The latter quantity is the mean value of the two principal curvatures.) 
[7] This assumes that the future apparent horizon $\mathcal{A}$ has the structure of a sufficiently smooth manifold. See, e.g., Proposition 12.2.5 of Ref. 柾.

[8] K. Thorne in Magic without Magic: John Archibald Wheeler, edited by J. R. Klauder (W. H. Freeman, San Francisco, 1972). It should be remembered that the imprecision in Thorne's hoop conjecture is intentional; making it precise is part of the problem. [What is meant by $M$ ? What is meant by a hoop encircling the body? Is the horizon a black-hole event horizon or a (future) apparent horizon?]

[9] E. Flanagan, Phys. Rev. D 442409 (1991); 461429 (1992).

[10] R. Schoen and S. -T. Yau, Commun. Math. Phys. 90, 575 (1983).

[11] P. Bizoń, E. Malec, and N. Ó Murchadha, Phys. Rev. Lett. 61, 1447 (1988); P. Bizoń, E. Malec, and N. Ó Murchadha, Class. Quantum Grav. 6, 961 (1989); T. Zannias, Phys. Rev. D 45, 2998 (1992).

[12] R. Schoen and S. -T. Yau, Commun. Math. Phys. 79, 231 (1981).

[13] D. M. Eardley, J. Math. Phys. 36, 3004 (1995).

[14] D. M. Eardley, Ann. N.Y. Acad. Sci. 688, 408 (1993).

[15] We call a tensor $h_{b}^{a}$, acting on a vector space $V$ with metric $g_{a b}$, a rank 2 orthogonal projection map if $h_{a b}$ is symmetric, $h_{b}^{a}=h^{a}{ }_{c} h_{b}^{c}$, and $h^{a}{ }_{a}=2$, where indices are raised and lowered with $g_{a b}$. The trace condition on $h^{a}{ }_{b}$ ensures that $h_{b}^{a}$ maps $V$ onto a two-dimensional vector subspace of itself, i.e., its rank is two. It is not difficult to show that with $\operatorname{dim}(V)=3$, there exists a unique (up to sign) unit vector $n^{a}$ such that $h^{a b}=g^{a b}-n^{a} n^{b}$. Further, any such unit vector $n^{a}$ defines a unique rank 2 orthogonal projection map in this way. While perhaps more complicated than it could be otherwise, this statement of the theorem follows the spirit of its proof.

[16] Y. Choquet-Bruhat, C. DeWitt-Morette, and M. DillardBleick, Analysis, Manifolds and Physics, Part I: Basics (North Holland, New York, 1989).

[17] R. Osserman, A Survey of Minimal Surfaces (Dover Publications, Inc., New York, 1986).

[18] H. Federer, Geometric Measure Theory (Springer-Verlag, New York, 1969). See also F. Morgan, Geometric Measure Theory: A Beginner's Guide (Academic Press, San Diego, 1988) and F. J. Almgren, Jr., Plateau's Problem: An Invitation to Varifold Geometry (W. A. Benjamin Inc., New York, 1966). 